\title{
Diseño de una lúdica para la asignatura de Gestión Financiera Enfocada en la Industria 4.0
}

Design of a game for the course of Gestión Financiera Enfocada en la Industria 4.0

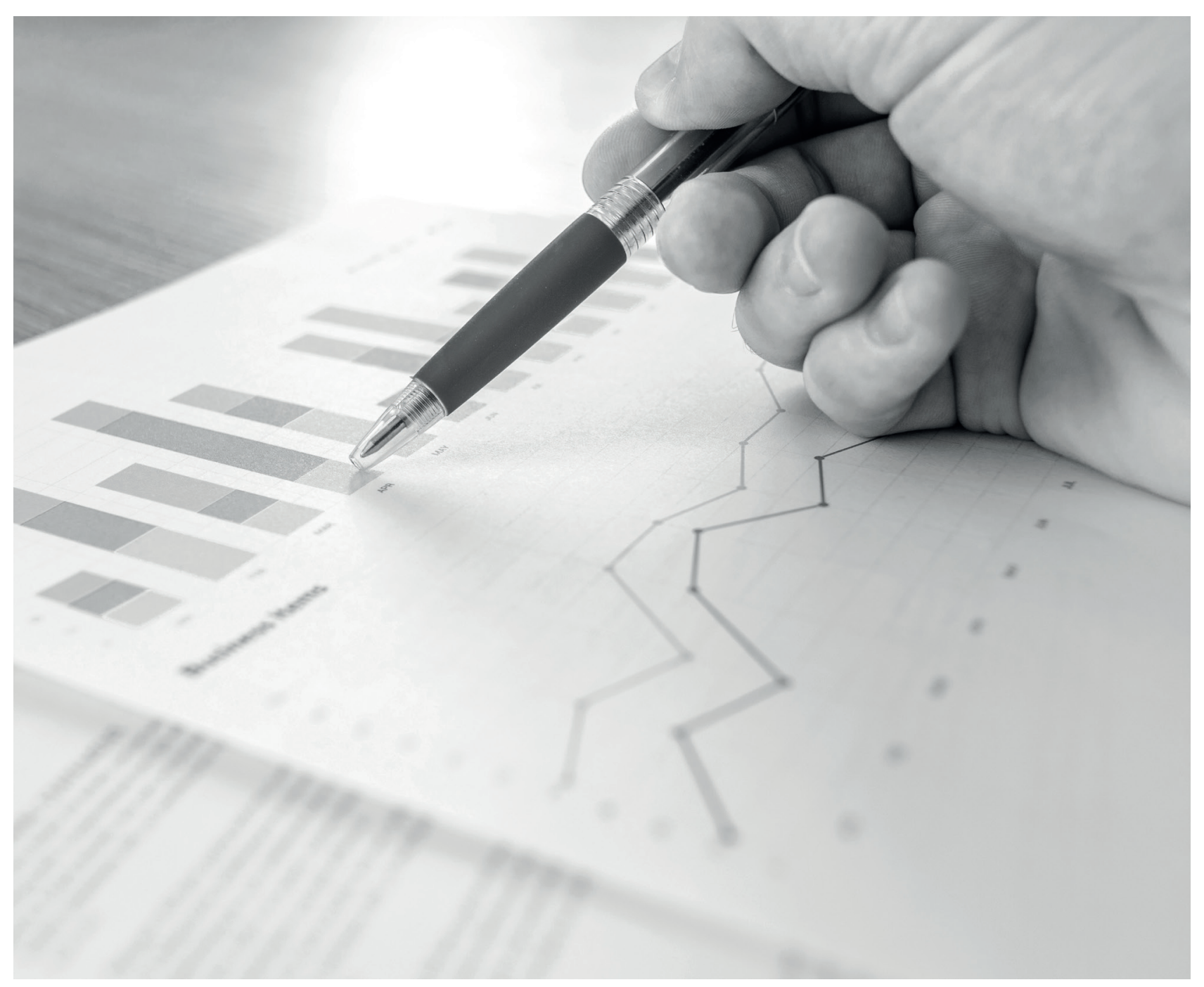




\title{
Diseño de una lúdica para la asignatura de Gestión Financiera Enfocada en la Industria 4.0 $0^{1}$
}

\author{
Design of a game for the course of Gestión Financiera Enfocada \\ en la Industria 4.0
}

\author{
Sarha Melissa Chica Otalvaro²
}

Artículo recibido en abril 27 de año 2020; artículo aceptado en septiembre 01 de 2020

\begin{abstract}
Este artículo puede compartirse bajo la Licencia Creative Commons Atribución-NoComercial-Compartirlgual 4.0 Internacional y se referencia usando el siguiente formato: Chica, S. (2021). Diseño de una lúdica para la asignatura de Gestión Financiera Enfocada en la Industria 4.0. I+D Revista de Investigaciones, 16(1), 136-146. http://dx.doi.org/10.33304/revinv.v16n1-2021012.
\end{abstract}

\begin{abstract}
Resumen
En los últimos años, las lúdicas se han convertido en herramientas didácticas que le permiten al docente complementar sus clases magistrales. Estas han sido de gran utilidad para evidenciar la apropiación del conocimiento por medio de la simulación en un contexto específico, en el cual los estudiantes deben aplicar componentes teóricos en la práctica. Dada la importancia de esta temática, la Facultad de Ingenierías de una institución universitaria de la ciudad de Armenia, Quindío, se dio a la tarea de fortalecer este tipo de aprendizaje práctico, a través de la creación y posterior validación de una lúdica enfocada en la gestión financiera y en el impacto de la cuarta revolución industrial a nivel empresarial. El diseño de la herramienta pedagógica se centra en los temas básicos asociados a las finanzas de las empresas que pretenden iniciarse en el mundo de la digitalización y las fábricas inteligentes. A lo largo de la lúdica, el estudiante detecta las competencias que debe desarrollar un ingeniero industrial para enfrentarse a los retos de la industria 4.0 asociados a las finanzas. Con base en esto, se realiza una evaluación donde se contrasta la lúdica como herramientas pedagógicas frente a la clase magistral, y se obtiene como resultado un aumento aproximado del $20 \%$ en la compresión de conceptos, de acuerdo con lo evaluado.
\end{abstract}

Palabras clave: Automatización, competencias, finanzas, industria, lúdicas.

\begin{abstract}
In the last years, playful games have become didactic tools that allows professors to complement their master classes. These have been very useful to demonstrate the appropriation of knowledge through the simulation of a specific context, in which students must apply theoretical components in practice. Given the importance of this theme, the Faculty of Engineering of the Corporación Universitaria Empresarial Alexander von Humboldt, set about strengthening this type of practical learning through the creation and subsequent validation of a game focused on financial management and the impact of the fourth industrial revolution at the business level. The design of the didactic tool focuses on the basic topics associated with the finances of companies that intend to start in the

\footnotetext{
${ }^{1}$ Artículo de investigación, con enfoque cualitativo, resultado de un proyecto de investigación en curso, perteneciente al área de ciencias sociales e ingeniería industrial, subárea de finanzas. Desarrollado en el Grupo de Investigación Eje Cafetero, Ciencia, Ingeniería e Industria, fue financiado por la Universidad Alexander Von Humboldt (CUE) (Armenia, Quindío). Dirección: avenida Bolívar n. ${ }^{1-189, ~ P B X: ~(6) ~ 7450025 . ~ F e c h a ~ d e ~ i n i c i o: ~ e n e r o ~}$ de 2019. Fecha de terminación: julio de 2020.

${ }^{2}$ Ingeniera Industrial de la Universidad Alexander Von Humboldt. Vinculada al Grupo de Investigación Eje Cafetero, Ciencia, Ingeniería e Industria, Universidad Alexander Von Humboldt (CUE) (Armenia, Quindío). Dirección: avenida Bolívar n. ${ }^{\circ}$ 1-189, PBX: (6) 7450025. ORCID ID: https://orcid. org/0000-0002-2662-9515 Correo electrónico institucional: schica4418@cue.edu.co
} 
Sarha Melissa Chica Otalvaro

Diseño de una lúdica para la asignatura de Gestión Financiera Enfocada en la Industria 4.0

world of digitization and smart factories, supported by the engineering laboratory equipment of the von Humboldt University(Calliope and Fischertechnik). Throughout the play, the student manages to detect the competencies that an industrial engineer must develop to face the challenges of Industry 4.0, and based on this, the tool is validated by means of a test applied to the participants where the impact of playful activity versus master class is compared.

Keywords: Finance, industry, automation, games, competitions.

\section{Introducción}

En el contexto académico actual, es importante contar con herramientas didácticas que le permitan al estudiante apropiarse de conceptos teóricos vistos en clase. Existen autores que apoyan la idea de que los juegos no son simplemente actividades que generan placer o diversión en los participantes (Echeverri y Gómez, 2009), sino que, al ser un agente activo del juego, se desarrollan competencias para vivir en sociedad. Esta última aclaración permite enlazar los juegos con la academia (Chacón, 2008), abordado desde las hoy conocidas estrategias lúdicas, una herramienta que ofrece un escenario en el cual se simula un contexto empresarial y permite evaluar competencias (del ser y del hacer) que normalmente en una clase magistral no se desarrollan (Argudín, 2001).

Los pioneros en Colombia de esta iniciativa para la creación de actividades lúdicas en el aula de clase fueron los participantes del grupo de investigación GEIO en la Universidad Tecnológica de Pereira, a partir del año 1999 (Grupo GEIO, 2020). Este grupo de personas se han dedicado desde entonces a desarrollar lúdicas con el fin de transferir conocimientos y potenciar habilidades claves en el ejercicio de la ingeniería industrial y sus áreas afines. Debido a los óptimos resultados obtenidos en el desarrollo de este ejercicio, nació la Red para la investigación, el desarrollo y la divulgación de procesos de enseñanza y aprendizaje. En esta comunidad se encuentran aproximadamente 26 universidades del país, las cuales se dedican al desarrollo de actividades lúdicas, con base en las competencias a desarrollar en las distintas disciplinas de la Ingeniería Industrial (López y Mejía, 2017).

Dado lo anterior, y considerando la importancia que tienen las lúdicas en el contexto académico, la extensión que estas han tenido en otras áreas de conocimiento y la importancia de lograr un cambio en la educación tradicional, surge la idea de fortalecer el programa de Ingeniería Industrial de una institución de educación superior de la ciudad de Armenia, con este tipo de metodología. Es así que se propone la creación de una lúdica (estrategias de aprendizaje por medio de la gamificación) que permita complementar los conceptos adquiridos en el aula de clase.

Por tanto, este articulo presenta el diseño y la validación de una lúdica que busca brindar un espacio a los estudiantes para que apliquen los conceptos y la parte teórica vista en clase en un contexto real (simulado), y así lograr que el individuo no solo aprenda viendo (AcostaMedina et al., 2020), sino haciendo.

\section{Marco referencial}

En el contexto laboral se habla frecuentemente de lo que es la industria 4.0, o, en su defecto, de la cuarta revolución industrial, que está llegando gradualmente al día a día de los individuos. Este fenómeno de tecnología e información supone un cambio abrupto en el concepto de industria que se conoce actualmente, pero, en realidad, ¿a qué hace referencia el término de industria 4.0? (Del Val Román, 2016).

Durante los últimos cuatro siglos, la humanidad ha presentado grandes avances que han cambiado la forma de convivir, y conforme a esto las industrias y sus sistemas económicos también se vieron afectados. En un primer momento, a mediados del siglo XVIII y principios del siglo XIX, se dispara lo que se conoce como primera revolución industrial (Cancela, 2001), la cual se caracteriza principalmente por la mecanización de los procesos de producción de la época, así como la aparición de una sociedad agrícola mucho más sedentaria.

Un siglo después, gracias al aumento en los niveles de educación y cultura, la humanidad logra alcanzar un grado mayor de evolución, que posteriormente se conocería como segunda revolución industrial. Las características principales de esta se enfocaron en el crecimiento de las empresas. Se adopta la electricidad como fuente de energía, lo que desencadena un sinfín de aplicaciones a nivel industrial, la aparición y aplicación de los buques movidos a vapor, así como las locomotoras, el avance en la industria química, eléctrica, de petróleo y de acero. Sin embargo, se afirma que la segunda revolución industrial fue un avance de la primera, ya que nunca se encuentra una ruptura entre ambas, ni un cambio de alto impacto en sus características; en ambas, se habla sobre el fortalecimiento de las industrias a través de la aplicación de sistemas y tecnologías.

A finales del siglo $X X$, se desarrolló la tercera revolución industrial. Es aquí donde se empieza a trabajar en distintos tipos de sistemas tecnológicos que logran cambiar totalmente la manera de ver el mundo y su funcionamiento en las distintas áreas (Gómez Suta et al., 2016). La característica principal de este periodo fue la implementación de la electrónica y la automatización de la industria, lo que permitió disminuir algunas 
actividades repetitivas que se daban dentro de las líneas de producción.

Después de dos décadas de haberse efectuado la tercera revolución industrial, los avances en cuanto a tecnología e innovación fueron creciendo de manera exponencial, lo que lleva a hablar actualmente de industria 4.0. La característica principal de esta revolución se centra en la implementación de la robótica y de componentes sensóricos. Es así como actualmente se habla de distintos conceptos relacionados con el internet de las cosas, internet de los servicios, big data, análisis avanzado de datos estadísticos, sistemas ciberfísicos, máquinas inteligentes, fábricas inteligentes, entre otros. La relación entre la electrónica y el manejo de las redes y la información ha logrado que se perciba un cambio a futuro respecto a la forma de vivir y de interactuar con el medio que se conoce hoy en día (Del Val Román, 2016).

En la literatura se encuentran distintas definiciones asociadas a la industria 4.0, pero todas nacen bajo la misma base. En efecto, la cuarta revolución industrial, que trae consigo las fábricas inteligentes, se fundamenta en la idea de la digitalización de la información y del manejo de esta a través de la interacción de las cosas (objetos, máquinas, etc.), como si fuese un sistema nervioso creado alrededor del mundo por las redes que se conectan entre sí y la información que fluye por estas. Es así como ya no se habla de una interacción de los seres humanos con el internet y la información, sino del internet directamente relacionado con aquellas cosas que más utilizan los individuos en el día a día, de manera que la información que quede almacenada en esta red permita facilitar las tareas aplicadas en cualquier ambiente. De esta forma, las industrias y las líneas de producción estarían en la capacidad de lograr aumentar su grado de flexibilidad en la creación de nuevos productos y servicios (Ynzunza et al., 2017).

Las industrias que más se han fortalecido gracias a la llegada y al nivel de adaptación que han tenido estos sistemas modernos son aeronáutica, automovilismo, energía, sector salud, entre otros. Estas industrias ya están aplicando la fabricación por adición, que se refiere a la producción realizada con material 3D (Ynzunza et al., 2017). La implementación de este tipo de tecnologías, además de perfeccionar las técnicas e indicadores de productividad, aumenta la calidad del producto final, lo que permite obtener revisiones eficientes del producto.

Por tanto, las industrias 4.0 prometen grandes cambios en la manufactura y producción de bienes y servicios que se conoce hoy en día, a través de la interacción de dispositivos inteligentes (inteligencia artificial). La pregunta a continuación sería qué competencias deberá desarrollar un profesional para desempeñarse eficientemente en dicha industria (Mantilla, 2018).
El tema a nivel de competencias para los nuevos y antiguos profesionales ha sido de gran apreciación. Incluso se puede hablar de la revolución de las competencias, ya que el profesional debe ir formándose de acuerdo con las necesidades del medio. Al estar en un entorno tan cambiante, es importante definir aquellas habilidades que deberá desarrollar el individuo para lograr sobrevivir en un entorno industrial competitivo (Terrés et al., 2017).

En este orden de ideas, es sumamente importante identificar los pilares de esta revolución, que se convertirán en los actores del cambio a nivel de industria. Como primera medida se resalta la importancia de los datos y la forma de recolectarlos. Como bien se habló en párrafos anteriores, la información será obtenida a través de la interacción que tiene el medio con los objetos sensóricos (internet de las cosas); así mismo, la manera en que se irá fortaleciendo el sistema nervioso mundial a través de la consolidación de los datos y la información. Adicionalmente, debe existir un sistema que sea capaz de analizar la información, así como las decisiones tomadas con base en ésta. Para esto, usualmente se habla de tecnologías como data analytics y machine learnning (Mantilla, 2018).

También se habla de un pilar con gran renombre, y tiene que ver con la simplificación de las tareas a través de la incorporación de las máquinas o de la realidad virtual. En este mismo orden de ideas, se resalta la importancia de establecer procedimientos que vayan de lo físico a lo digital y de lo digital a lo físico. Es decir, que se identifique la necesidad del medio físico, se lleve a una simulación y posteriormente pueda crear una solución a través de plataformas digitales para lograr adaptarla a la realidad.

Estos pilares y características mencionados anteriormente tienen mucha relación con las competencias que debe desarrollar un individuo para enfrentarse a los cambios que trae consigo la cuarta revolución industrial.

En primer lugar, como características principales a desarrollar, se reconoce el tipo de liderazgo que debe tener un profesional que gestione procesos enfocados en la industria 4.0, quien debe enfocarse principalmente en el desarrollo de las competencias de gestión; es decir, la capacidad de optimizar procesos de mejora, así como en fortalecer el nivel de sus competencias técnicas. Así mismo, cabe resaltar la importancia del trabajo en equipo sin perder el componente humano (Del Val Román, 2016).

Las competencias del ingeniero en el entorno actual deben centrarse en la autonomía y las mejoras en la toma de sus decisiones, reconociendo la importancia del aprendizaje continuo y el aumento exponencial del grado de especialización en su trabajo.

Con la descripción anterior acerca de las tecnologías que engloba la cuarta revolución industrial, es de suma importancia articular el cambio industrial con el impacto 
que este tendría en las empresas a nivel financiero, y este es precisamente el tema por abordar dentro de la herramienta didáctica que se desea construir.

Desde las competencias blandas se espera fortalecer toda la parte actitudinal y de liderazgo que debe ser propia del ingeniero en formación. Se enfatiza en la importancia de reconocer la función de las finanzas en la industria 4.0. Este concepto se conoce también como finanzas 4.0, y se refiere a la interacción que debe realizar el área de finanzas en una compañía con la digitalización de los datos, y a partir de esto reconocer lo que le espera a la organización y las inversiones a mediano y largo plazo en cuanto a tecnología (Ynzunza et al., 2017).

En el ámbito empresarial la gestión financiera es considerada como uno de los aspectos fundamentales en las organizaciones, ya que esta área del conocimiento permite realizar una efectiva administración de los recursos de la organización, de manera que la empresa pueda gestionar sus ingresos y egresos de forma óptima (Escribano, 2011). Así mismo, los directivos deben tomar decisiones que beneficien a la organización en un determinado plazo.

Dentro de los elementos básicos referentes a la administración o gestión financiera, se pueden encontrar los principios financieros básicos basados en una experiencial gerencial (Peŕez-Carballo Veiga, 2015), la innovación financiera de productos y del mercado, el desarrollo tecnológico de los sistemas de gestión y comunicación $y$, finalmente, las oportunidades y amenazas del entorno (Escribano, 2011).

La gestión financiera para el programa de Ingeniería Industrial de la Corporación Universitaria Empresarial Alexander Von Humboldt tiene como objetivo desarrollar en el estudiante las siguientes competencias (CUE Alexander Von Humboldt, 2018):

- Reconocer los presupuestos y las proyecciones como fuente de datos para diversos propósitos, como la planeación, el control, la información y costeo de productos y servicios, líneas de productos y clientes, sin limitarse solo a procedimientos, sino incluyendo conceptos y análisis para que la contabilidad de los costos sea una valiosa herramienta administrativa para todas las empresas.

- Reconocer la importancia del presupuesto y el control presupuestario como herramientas de planificación y control.

- Analizar los distintos presupuestos y su gravitación dentro del esquema operativo de la empresa.

- Comprender las técnicas para evaluar el costo de la unidad producida, tanto de bienes como de servicios.

- Identificar los sistemas de proyección y su aplicación en empresas comerciales, industriales y de servicios.

- Comprender cómo las proyecciones pueden facilitar la planeación, el control y la toma de decisiones.

\section{Metodología}

Esta investigación es descriptiva y cualitativa, ya que la recolección de información está basada en comportamientos naturales y respuestas abiertas que requieren una posterior interpretación. El tipo de estudio es experimental, pues consiste en el diseño de una herramienta de aprendizaje gamificada y, posterior al diseño, se realiza una evaluación del impacto que tiene la lúdica frente a las clases magistrales.

Para el desarrollo de la estructura a nivel documental de la lúdica, se tomó como base el trabajo propuesto por Gómez (2010), en el cual se plantea una metodología clara para la construcción de lúdicas enfocadas en una temática específica. Adicionalmente, se involucró un componente esencial en el diseño de la herramienta pedagógica, basada en la metodología design thinking, la cual se ajusta de manera adecuada a las necesidades que se presentan hoy por hoy en el contexto académico (Flores y Tena, 2016).

Esta metodología consta de 5 pasos fundamentales para la puesta en marcha de proyectos, que vincula a los actores relevantes del proceso en la búsqueda de una solución (Brown, 2008). En primer lugar, se busca empatizar, con el fin de lograr que las personas involucradas se sientan realmente parte del proceso de construcción de la solución. Para el proyecto en cuestión, se consolida la temática de la lúdica con personal docente experto en el área. En segundo y tercer lugar se encuentra la definición del problema y la búsqueda de la solución, respectivamente. Por medio de la aplicación de estos pasos al desarrollo del proyecto, se centra en la identificación de la competencia a fortalecer, y la respuesta a esto estaría dada por la lúdica construida. Posteriormente, se habla del testeo y la evaluación, asociados a las pruebas piloto para la verificación de la solución propuesta. Lo anterior se ve reflejado en el ejercicio de validación de la lúdica y el impacto como estrategia pedagógica en el aula de clase.

\section{Resultado}

- Primera fase:

En esta fase se relaciona el proceso de diseño de la lúdica, donde se explican los parámetros para la selección de la temática a desarrollar y la elaboración de la estructura referente al procedimiento a seguir para la ejecución de la lúdica.

La selección de la temática se realizó a través de la convocatoria de un panel de expertos, en el cual participaron docentes de una institución universitaria en Armenia, Quindío, los cuales han tenido más de 5 años de experiencia en la docencia asociada al tema de finanzas y contabilidad. Durante esta sesión, se analizó el microcurrículo de la asignatura de gestión financiera, 
haciendo énfasis en las competencias que el estudiante debería desarrollar, las cuales se mencionaron en el capítulo anterior del presente artículo. En el desarrollo del panel de expertos, los docentes encontraron la necesidad de fortalecer las temáticas asociadas a las competencias que un ingeniero industrial debe adquirir para enfrentarse a los retos de la cuarta revolución industrial desde una perspectiva financiera, y de responder a las variables asociadas con la inversión en maquinaria para la automatización de procesos y la transformación digital dentro de las empresas. De este análisis se construyeron los objetivos que enmarcan la lúdica, así como el test para validar las competencias que cumplen con dichos objetivos (el cual se mencionará en el ejercicio de validación). Posteriormente se inició el proceso de diseño de la guía, donde se expone el paso a paso de las actividades, los recursos necesarios para su desarrollo, las reglas y los demás componentes fundamentales que permiten la óptima implementación de la lúdica en el aula de clase, los cuales se detallan a continuación.

Las competencias que se esperan fortalecer con la lúdica son:

- Análisis financiero para una empresa que requiera ejecutar una transformación digital en sus procesos.

- Análisis comparativo de los procesos manuales y automatizados, referente a una línea de producción.

Al hablar de finanzas 4.0, se refleja la intención de digitalizar los procesos financieros dentro de una empresa, para lograr obtener un incremento en la calidad de la información financiera, referente a los informes, a los indicadores y la organización de los datos. Esto con el fin de aumentar la simplicidad en los procesos, sin restarle importancia al área financiera dentro de una organización (Escribano, 2011). Sin embargo, en la lúdica diseñada se espera que los estudiantes logren apreciar los cambios y las decisiones que deben tomar a nivel financiero, en cuanto a inversión en maquinaria, en capacitaciones y otras variables que involucren la innovación tecnológica dentro de los procesos.

- Nombre de la lúdica: Un acercamiento a las finanzas 4.0

- Objetivos:

- Realizar el análisis financiero para una empresa que requiera ejecutar una transformación digital en sus procesos.

- Identificar las competencias que un ingeniero industrial debe desarrollar para enfrentarse con la cuarta revolución industrial.

- Duración: 4-5 horas.

- Recurso humano: 10 o 12 personas (participantes y
1 o 2 coordinadores de la lúdica.

- Recursos materiales:

- Carros Fischertechnik (cantidad: 40)

- Calliope Mini, kits fischertechnik

- Hojas de papel

- Plantilla de presupuestos e indicadores

- Guía de programación ventilador

- Computadores portátiles

- Video beam

- 5 mesas grandes

- 14 sillas

\section{Estructura de la lúdica}

La lúdica gira alrededor de la fabricación de automóviles, y se desarrollará a lo largo de 3 periodos (pueden considerarse como años o semestres); por lo tanto, estará dividida en 3 momentos de dos horas cada uno, aproximadamente. Inicialmente, los estudiantes deberán construir los presupuestos asociados con la empresa dedicada a la fabricación de automóviles y proyectarlos a lo largo de los 3 años siguientes, considerando los parámetros para la construcción de estos. Aquí se debe tener en cuenta la mano de obra (la construcción de la línea de producción), los gastos y costos involucrados, los inventarios de materia prima e insumo, entre otros.

Transcurrido un año, los participantes deberán construir una propuesta para la automatización de una parte del proceso productivo, teniendo en cuenta la inversión de la maquinaria, los cambios que influirían en el proceso y cómo impactaría financieramente este cambio en la empresa a nivel financiero. Esto se deberá realizar a lo largo de cada periodo para determinarlo con los indicadores financieros pertinentes, de manera que se contemple el estado de la empresa, periodo tras periodo.

\section{Reglas}

Para el primer periodo, deberán establecer mínimo 4 estaciones de trabajo para la elaboración del automóvil, teniendo en cuenta que hay una estación adicional encargada de realizar la actividad del acabado de pintura, que tarda alrededor de 1,2 minutos para realizarse.

Los participantes de la lúdica deberán ubicar sus estaciones de trabajo del área de producción en la banda transportadora del laboratorio, diseñando las actividades que le corresponderán a cada operario.

Los estudiantes tendrán que averiguar previamente la información relacionada con los gastos y costos involucrados dentro del ejercicio de la empresa. Estos datos deberán ser reales (información obtenida por medio de los funcionarios de la universidad) y tendrán que delegar a una persona para poder recolectarlos, de manera que no se generen reprocesos. De esta forma, podrán realizar el diligenciamiento de los presupuestos. 
Sarha Melissa Chica Otalvaro

Diseño de una lúdica para la asignatura de Gestión Financiera Enfocada en la Industria 4.0

Etapa inicial: Socialización de reglas, explicación de la lúdica

Se explicará de forma clara y precisa el objetivo de la lúdica, los diferentes momentos que tendrá y las reglas involucradas. Los estudiantes serán los encargados de construir el presupuesto del año inicial y proyectarlo a lo largo de 3 años. Los presupuestos a construir serán los siguientes:

- Ventas

- Producción

- Compra de materia prima

- Presupuesto de mano de obra

- Presupuesto de CIF

- Presupuesto de gastos administrativos

- Presupuesto de inversión

- Estado de costo de ventas

- Estado de resultados

- Flujo de caja

- Estado de situación financiera

Los participantes deben construir cada una de las estaciones de trabajo, determinando la actividad correspondiente a la elaboración del automóvil, y deberán realizar una toma de tiempos con la que puedan determinar la capacidad de la línea, los costos de producción involucrados y los indicadores de producción que deseen para medir su efectividad.

\section{Etapa intermedia}

En esta etapa los estudiantes deberán elegir una propuesta de automatización para el proceso, con el fin de determinar los costos involucrados y el impacto que tendrá a nivel productivo. Un externo (asistente/monitor de laboratorios), quien representará el proveedor de la empresa de transformación tecnológica, será el encargado de promocionar el producto, mediante el cual podrán implementar una propuesta de automatización a su proceso, detallando sus características y su precio.

El producto por promocionar será un ventilador que disminuirá el proceso de secado en la estación de pintura. El mecanismo del ventilador se presenta en la Figura 1.

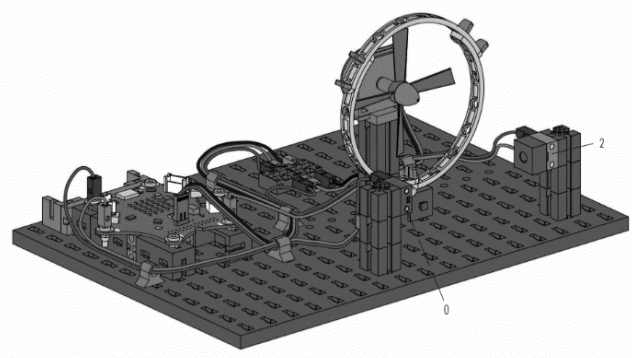

Figura 1. Diagrama Mecanismo Ventilador. Fuente: Fischertechnik (2020).
De igual forma, los líderes de la compañía deberán contemplar la viabilidad de la inversión y tener en cuenta la capacitación que debe ser pagada para que logren darle un correcto uso al equipo.

La capacitación recibida por parte del proveedor externo se realizará teniendo en cuenta la programación de la máquina, donde la empresa deberá elegir a dos de sus integrantes para ser capacitados. En primer lugar, se hará una breve introducción a la plataforma que se utilizará, denominada Open Roberta y, a partir de esto, se explicará la forma en la cual debe programarse el mecanismo para su correcto funcionamiento. Al grupo también se le entregara una guía de armado para lograr ensamblar la máquina. En la Figura 2 se presenta la programación final que debería tener el prototipo.

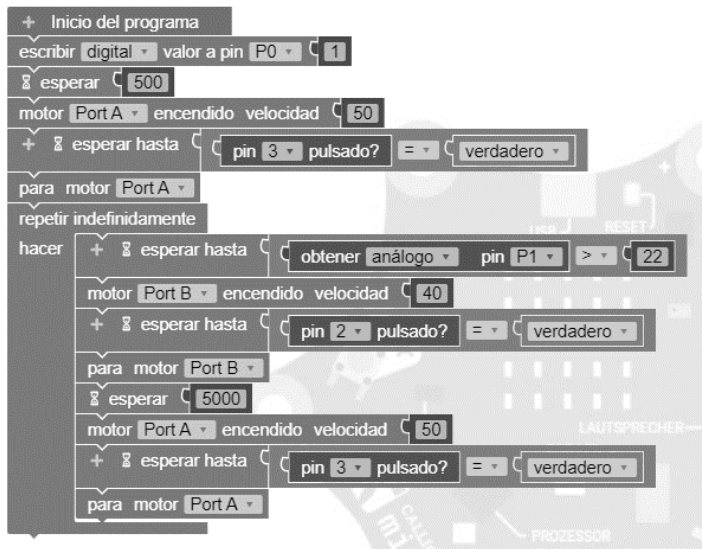

Figura 2. Programación mecanismo ventilador. Fuente: Plataforma Open Roberta.

Posterior a este momento de la lúdica, el grupo estará en la capacidad de programar y montar de manera eficiente el mecanismo para su correcto funcionamiento. En esta etapa, el grupo también podrá realizar las mejoras que considere pertinentes a nivel de proceso, teniendo en cuenta que la regla de mínimo 4 estaciones desaparece, pero la producción deberá realizarse en serie. A continuación, deberá realizar nuevamente una toma de tiempos, en la cual logren contemplar los cambios dentro del proceso y el impacto que tiene este cambio en sus indicadores.

\section{Etapa final}

Este será el espacio destinado para las conclusiones del ejercicio y la socialización de las competencias que identificaron los estudiantes a lo largo de la lúdica, propias de un ingeniero industrial en un contexto de industria 4.0. Esta adquisición de conocimiento se evaluará por medio de un test aplicado a los estudiantes, con el fin de realizar el ejercicio de validación el cual se describe a continuación. 


\section{- Segunda fase:}

Se desarrolla la segunda fase relacionada con la validación de la lúdica, a través de un análisis comparativo entre la apropiación de conocimiento por parte de los estudiantes, mediante una clase magistral y la apropiación de conocimiento a partir de la lúdica propuesta.

El instrumento construido para la validación se aplicó a los estudiantes de sexto semestre del programa de Ingeniería Industrial, entre las edades de 20 y 24 años, en dos momentos y grupos diferentes.

La validación descrita a continuación se divide en dos momentos.

Inicialmente se implementó el test a los estudiantes que habían recibido la clase magistral asociada a las competencias que debería desarrollar un ingeniero industrial para enfrentarse a las Fábricas Inteligentes (momento 1). Como estos estudiantes ya habían recibido una clase referente al tema, se esperó un semestre para poder ejecutar la lúdica con estudiantes distintos que estuvieran en el mismo semestre (momento 2 ), de manera que se presentara homogeneidad en los grupos en cuanto a su nivel académico.

A continuación, se presenta el instrumento validado por los integrantes del panel de expertos y los resultados obtenidos en la primera fase; además, se anexa el instrumento con la justificación de las respuestas correctas.

1. ¿Qué características tendrá el estilo de liderazgo que requerirán las organizaciones 4.0 ?

a) Liderazgo, organización, competencias técnicas, autonomía, desarrollo de proyectos.

b) Trabajo en equipo, cualidades humanas, competencia de gestión, formación y desarrollo.

c) Competencias técnicas de mando, capacidad de gestión de un proyecto de mejora, cualidades humanas, liderazgo.

R/ B. Justificación: Las competencias esenciales para un líder en un entorno 4.0 serán el trabajo en equipo, la capacidad de relacionarse y las competencias de gestión, las cuales integran conocimientos técnicos con la capacidad de gestionar proyectos de mejora, así como la capacidad de inspirar el crecimiento y la formación de los colaboradores en el interior de la empresa. Al ser coherentes con las características de las empresas 4.0, los operarios tendrán que obtener un grado de especialización mayor en su tarea.
2. ¿Cuáles considera que serán las competencias 4.0 que deberán desarrollar los trabajadores en una organización?

a) Autonomía, capacidad para tomar decisiones, aumento en el grado de especialización en su trabajo, aprendizaje continuo.

b) Trabajo en equipo, capacidad de relacionarse con sus pares, proyección, aprendizaje continuo.

c) Capacidad para tomar decisiones, aprendizaje continuo, trabajo en equipo, relaciones interpersonales.

R/ A. Justificación: Dentro de las organizaciones 4.0 es primordial contar con personal autónomo, que tenga la capacidad de tomar decisiones de acuerdo con su criterio y experiencia. Esto se acompaña de un grado de especialización en su trabajo y el interés sobre ese aprendizaje continuo de sus labores, ya que las actividades operativas tienden a desaparecer.

3. ¿Cuál cree que será el reto a nivel financiero para una organización 4.0 ?

a) Desarrollar herramientas que aumenten la confianza de los clientes en la organización y que cierren las brechas de información entre las partes, con el fin de aumentar la transparencia en los procesos realizados en el interior de la empresa.

b) Integrar y analizar los datos y comportamientos de sus clientes para tomar decisiones y diseñar estrategias de negocios futuras, complementado lo anterior con proyectos de inversión en tecnología, de forma que se mejoren los indicadores de valoración en la empresa.

c) Adoptar las herramientas digitales (internet de las cosas, fabricación aditiva, big data) que les permita llevar a cabo proyectos que sean coherentes con la cuarta revolución industrial, aumentando la rentabilidad, así como los indicadores de valoración de la empresa.

R/ B. Justificación: La finalidad del área de finanzas en una organización 4.0 será tomar decisiones a través del análisis que contemple posibles estrategias de negocio a largo plazo.

4. ¿Cuál es el objetivo de las industrias 4.0?

a) Aumentar las competencias de los negocios con el desarrollo de estrategias de innovación y proyectos tecnológicos.

b) Implementar técnicas avanzadas de producción y operaciones con tecnologías inteligentes en las diferentes industrias.

c) Desarrollar proyectos de innovación para el crecimiento tecnológico y financiero de las organizaciones. 
R/ B. Justificación: Implementar técnicas avanzadas de producción y operaciones con tecnologías inteligentes en las diferentes industrias. El objetivo de la industria 4.0 es implementar habilitadores digitales dentro de las distintas tipologías de negocio.

5. ¿Cómo cree usted que debería modificarse la estructura curricular en las universidades para aportar a dichas organizaciones 4.0 ?

La pregunta 5 queda abierta a los comentarios y perspectiva del alumno.

\section{Discusión}

Como se describe en el apartado de validación del capítulo anterior, el instrumento para la validación fue aplicado en el Momento 1 a un grupo de estudiantes de sexto semestre del programa de Ingeniería Industrial (semestre en el cual se profundiza en las temáticas asociadas a las finanzas). Este grupo estaba conformado por 10 estudiantes entre las edades de 20 y 25 años. A cada uno de ellos se les socializó el objetivo de la investigación, así como el consentimiento informado mediante el cual aceptaban su participación en el proyecto de forma voluntaria.

Este grupo de estudiantes cursó la asignatura de Gestión Financiera bajo una metodología netamente magistral, y el tema asociado a las industrias 4.0 pasó de largo y se mencionó únicamente en una de las clases.

A continuación se muestran los resultados obtenidos al aplicar el test a los estudiantes en el Momento 1.

Tabla 1

Gráfico instrumento evaluativo Momento

\begin{tabular}{|l|c|c|c|}
\hline \multicolumn{4}{|c|}{ Momento 1 } \\
\hline Pregunta/Respuesta & A & B & C \\
\hline Pregunta 1 & 0,1 & $\mathbf{0 , 6}$ & 0,3 \\
\hline Pregunta 2 & $\mathbf{0 , 3}$ & 0,2 & 0,5 \\
\hline Pregunta 3 & 0 & $\mathbf{0 , 4}$ & 0,6 \\
\hline Pregunta 4 & 0,3 & $\mathbf{0 , 1}$ & 0,6 \\
\hline
\end{tabular}

\section{Respuesta correcta}

Fuente: Autores.

En la Tabla 1 se observa que la mayoría de los participantes tienen claras las competencias de liderazgo que debe presentar un profesional dirigido a la industria 4.0, ya que más del $50 \%$ del grupo respondió acertadamente la pregunta . $^{\circ} 1$. Sin embargo, hay una falencia en cuanto desconocimiento por parte del $70 \%$ de los encuestados en lo que respecta a las demás preguntas del instrumento.
Esto resalta aún más la importancia de generar espacios que suplan estas necesidades a nivel de formación.

En el segundo momento se ejecutó la validación a un grupo conformado por 8 estudiantes de sexto semestre del programa de Ingeniería Industrial, que aún no estaban relacionados con la temática propia de la lúdica. Se realizó el ejercicio de socialización del objetivo del proyecto y el consentimiento informado, para posteriormente ejecutar la corrida de la lúdica con los estudiantes voluntarios. Al final se aplicó el mismo instrumento para corroborar el impacto de la lúdica como herramienta pedagógica.

En la Tabla 2 se puede apreciar que aproximadamente el $80 \%$ del grupo respondió de manera acertada las preguntas del instrumento.

Tabla 2

Gráfico instrumento evaluativo Momento 2

\begin{tabular}{|l|c|c|c|}
\hline \multicolumn{4}{|c|}{ Momento 2 } \\
\hline Pregunta/Respu & A & B & C \\
\hline Pregunta 1 & 0,125 & $\mathbf{0 , 8 7 5}$ & 0 \\
\hline Pregunta 2 & $\mathbf{0 , 8 7 5}$ & 0,125 & 0 \\
\hline Pregunta 3 & 0 & $\mathbf{0 , 8 7 5}$ & 1 \\
\hline Pregunta 4 & 0 & $\mathbf{1}$ & 0 \\
\hline
\end{tabular}

\section{Respuesta correcta}

Fuente: Autores.

De esta forma se logra evidenciar el impacto que tiene la lúdica en la interiorización de competencias y conceptos. Al vivenciar los escenarios simulados en una temática específica, se identifica el aumento de satisfacción por parte de los estudiantes frente a la apropiación de conceptos respecto a la temática tratada. En la Tabla 3 se exponen los resultados comparativos de ambos momentos de validación.

Tabla 3

Momentos de validación

\begin{tabular}{|c|c|c|}
\hline \multicolumn{3}{|c|}{ Comparación } \\
\hline Pregunta & $\begin{array}{c}\text { \% de respuestas } \\
\text { correctas momento 1 }\end{array}$ & $\begin{array}{c}\text { \% de respuestas } \\
\text { correctas momento 2 }\end{array}$ \\
\hline Pregunta 1 & $60 \%$ & $87,50 \%$ \\
\hline Pregunta 2 & $30 \%$ & $87,50 \%$ \\
\hline Pregunta 3 & $40 \%$ & $87,50 \%$ \\
\hline Pregunta 4 & $10 \%$ & $100 \%$ \\
\hline
\end{tabular}

Fuente: Autores. 


\section{Conclusiones}

La lúdica construida apoyará las clases en temas referentes a la digitalización de las líneas de producción, involucrando las distintas variables que impacten fuertemente un proceso. De esta forma, los participantes estarán preparados para enfrentarse a entornos en los cuales se estén presentando síntomas iniciales del impacto de la cuarta revolución industrial desde las temáticas relacionadas con la inversión de maquinaria y capacitación de operarios (Barros, 2017).

Las actividades de enseñanza y aprendizaje lúdicas, que se abordan desde las aulas de clase, han tomado fuerza en los últimos años, debido a la aceptación que han tenido por la comunidad académica al evidenciar el desarrollo de competencias, a través de entornos simulados enfocados en una temática central.

La lúdica se centró en la evaluación de esta como herramienta didáctica y no a nivel estructural; sin embargo, se logró identificar que los tiempos de programación del mecanismo dentro de la línea de producción que se habían planteado en el diseño inicial de la lúdica no fueron adecuados, ya que los estudiantes se toman más tiempo en la ejecución de esta actividad. Esto sugiere una oportunidad de mejora de la lúdica antes de efectuar su implementación.

El desarrollo de esta lúdica, desde su proceso de construcción hasta su validación, permite obtener un primer borrador asociado a un procedimiento para evaluar y validar las lúdicas como herramientas pedagógicas. Es así un insumo que facilita la construcción de herramientas evaluativas (test, rúbricas) que validen los resultados de aprendizaje y/o competencias que logra desarrollar el estudiante que participa de la actividad.

\section{Referencias}

Acosta-Medina, J. K., Torres-Barreto, M. L., ÁlvarezMelgarejo, M. y Paba-Medina, M. C. (2020). Gamificación en el ámbito educativo: Un análisis bibliométrico. I+D Revista de Investigaciones, 15(1), 30-39. https://doi.org/10.33304/revinv.v15n12020003

Argudín, Y. (2001). Educación basada en competencias. Revista Magistralis, 20, 39-61.

Barros, T. (2017). La industria 4.0: Aplicaciones $e$ implicaciones. Universidad de Sevilla.

Brown, T. (2008). Design Thinking. Harvard Business Review, 86(6), 84.

Cancela, I. (2001). La Revolución Industrial. http://www. capacitacion.edu.uy/files/medios/cd_prosoc09/
sitio/lecturas/Modulo_1/La_Revolucion_Industrial. pdf

Chacón, P. (2008). El juego didáctico como estrategia de enseñanza y aprendizaje ¿Cómo crearlo en el aula? Nueva Aula Abierta, 16(5), 1-8.

CUE Alexander Von Humboldt. (2018). Proyecto educativo del programa Ingenieria industrial. https://www. cue.edu.co/admin/js/plugins/ckeditor/kcfinder/ upload/files/PEP.pdf

Del Val Román, J. L. (2016). Industria 4.0: la transformación digital de la industria. http://coddii.org/wp-content/ uploads/2016/10/Informe-CODDII-Industria4.0.pdf

Echeverri, J. H. y Gómez, J. G. (2009). Lo lúdico como componente de lo pedagógico, la cultura, el juego y la dimensión humana. https://blog.utp.edu.co/ areaderecreacionpcdyr/files/2012/07/LO-LUDICOCOMO-COMPONENTE-DE-LO-PEDAGOGICO.pdf

Escribano, G. (2011). Gestión financiera. Paraninfo. (3. ${ }^{\text {a ed.). }}$

Fischertechnik. (2020). Calliope. Programar en educación primaria. Fischertechnik. https://www. fischertechnik.de/es-es/productos/aprender/stemrobotics/544626-calliope

Flores, A. y Tena, R. (2016). Design Thinking : Educational Innovation and Methodological Research. Didáctica, Innovación y Multimedia, 33, 1-6.

Gómez, M. C. (2010). Definición de un método para el diseño de juegos orientados al desarrollo de habilidades gerenciales como estrategia de entrenamiento empresarial. http://www.bdigital.unal.edu. co/1968/1/32242923.20101.pdf

Gómez Suta, M., Zuluaga Ramírez, C. y Arenas Valencia, W. (2016). Propuestas de herramientas pedagógicas experienciales como instrumentos claves en la formación de ingenieros competentes para apoyar el proceso de enseñanza-aprendizaje del área de investigación de operaciones y estadística. https:// antiguo.acofipapers.org/index.php/eiei2016/2016/ paper/viewFile/1537/566

Grupo GEIO. (2020). Quiénes somos. https://academia.utp. edu.co/geio/nosotros-edicion-2/

López, D. C. y Mejía, L. A. (2017). Una mirada a las estrategias y técnicas didácticas en la educación en ingeniería. Caso Ingeniería Industrial en Colombia. Entre Ciencia e Ingeniería, 11(21), 123. https://doi. org/10.31908/19098367.3290 
Sarha Melissa Chica Otalvaro

Diseño de una lúdica para la asignatura de Gestión Financiera Enfocada en la Industria 4.0

Mantilla, S. (2018). La cuarta revolución industrial está aquí, ¿está usted preparado? file:///C:/Users/usuario/ Downloads/Revolución 4.0 142201.pdf

Open Roberta (s.f). Programa NEPOprog. https://lab. open-roberta.org/

Peŕez-Carballo Veiga, J. (2015). La gestión financiera en la empresa (1. ${ }^{a}$ ed.). ESIC Editorial.

Terrés, J. I., Lleó de Nalda, Á., Viles, E. y Santos, J. (2017). Competencias profesionales 4.0. file:///C:/Users/usuario/Downloads/ Competenciasprofesionales4.0.pdf

Ynzunza, C., Izar, J., Bocarando, J., Aguilar, F. y Larios, M. (2017). El entorno de la industria 4.0: Implicaciones y perspectivas futuras. ConCiencia Tecnológica, 54, 33-45. 